\title{
Research on Key Technologies of Network Centric System Distributed Target Track Fusion
}

\author{
Yi Mao, ${ }^{1,2}$ Yun-ru Li, ${ }^{2}$ and Jin-fu Zhu ${ }^{1}$ \\ ${ }^{1}$ College of Civil Aviation, Nanjing University of Aeronautics and Astronautics, 29 Yudao St., Nanjing, Jiangsu 210016, China \\ ${ }^{2}$ State Key Laboratory of Air Traffic Management System and Technology, 1 Yongzhi Road, Nanjing, Jiangsu 210000, China
}

Correspondence should be addressed to Yi Mao; mao_y@nuaa.edu.cn

Received 7 November 2016; Revised 5 January 2017; Accepted 7 February 2017; Published 6 March 2017

Academic Editor: Nazrul Islam

Copyright ( 2017 Yi Mao et al. This is an open access article distributed under the Creative Commons Attribution License, which permits unrestricted use, distribution, and reproduction in any medium, provided the original work is properly cited.

\begin{abstract}
To realize common tactical picture in network-centered system, this paper proposes a layered architecture for distributed information processing and a method for distributed track fusion on the basis of analyzing the characteristics of network-centered systems. Basing on the noncorrelation of three-dimensional measurement of surveillance and reconnaissance sensors under polar coordinates, it also puts forward an algorithm for evaluating track quality (TQ) using statistical decision theory. According to simulation results, the TQ value is associated with the measurement accuracy of sensors and the motion state of targets, which is well matched with the convergence process of tracking filters. Besides, the proposed algorithm has good reliability and timeliness in track quality evaluation.
\end{abstract}

\section{Introduction}

The rapid development and wide application of information technology have promoted the informatization of weapon equipment and the continuous innovation of military theories. Network Centric Warfare (NCW) is an inevitable product in information era. The essence of NCW is to organize combat power based on Common Operational Picture [1].

The modern warfare is not only the confrontation between two groups of people or weapon, and the leading position largely depends on the C4ISR system of both sides [2-4]. The C4ISR system has its own communication network to meet the requirements of command and control $(\mathrm{C} 2)[5,6]$. However, when a battlefield commander is confronted with some threats and targets that need quick reaction and realtime processing, it would be insufficient to simply rely on traditional communication networks [7-11]. The commander needs special means to quickly draw the tactical picture of the whole battlefield, and data link is the best choice among these means.

The main tactical application functionality of data-linknetwork-centered combat system includes situation picture sharing, electronic countermeasures, tactical coordination, command and control, etc. [12-14]. This paper mainly discusses the model and algorithm of distributed target track fusion to realize situation picture uniformity and sharing among all members in a data link network.

\section{Distributed Processing Environment Construction}

The construction of distributed track fusion processing environment is based on the characteristics of Link 16, which includes flat network structure, no center node within the network, self-supply of intelligence, limited network capacity, etc. [15].

Basing on the above-mentioned characteristics, a layered architecture for network centric system information processing was designed, which mainly built a common application service layer, providing bidirectional transparent service between application systems and data transmission. The architecture aims to construct high-speed and flexible interoperating environment for tactical community. Shared information pool is established through resource virtualization, distributed computing, and networked storage in the 


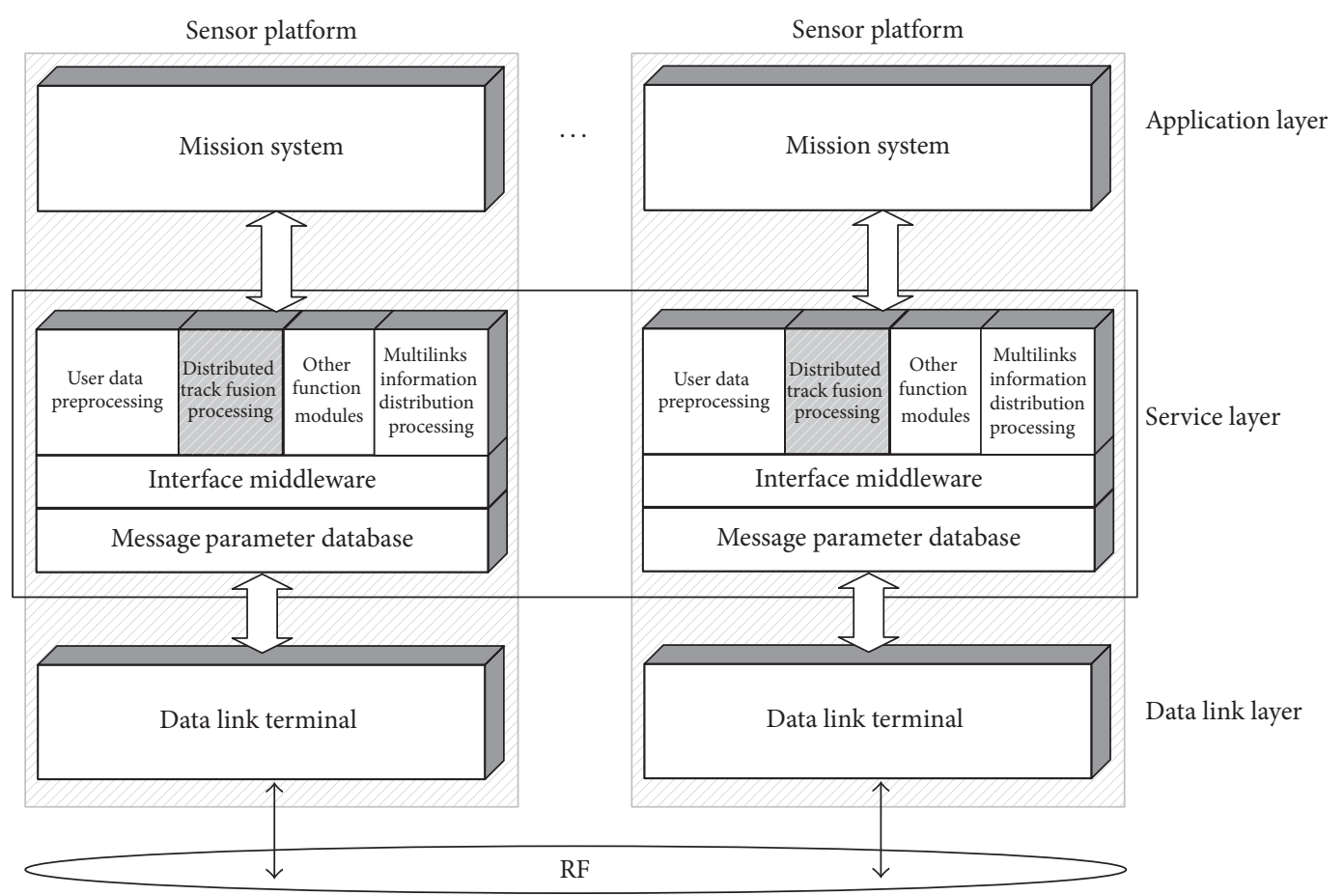

FIGURE 1: The layered architecture of network centric system information processing.

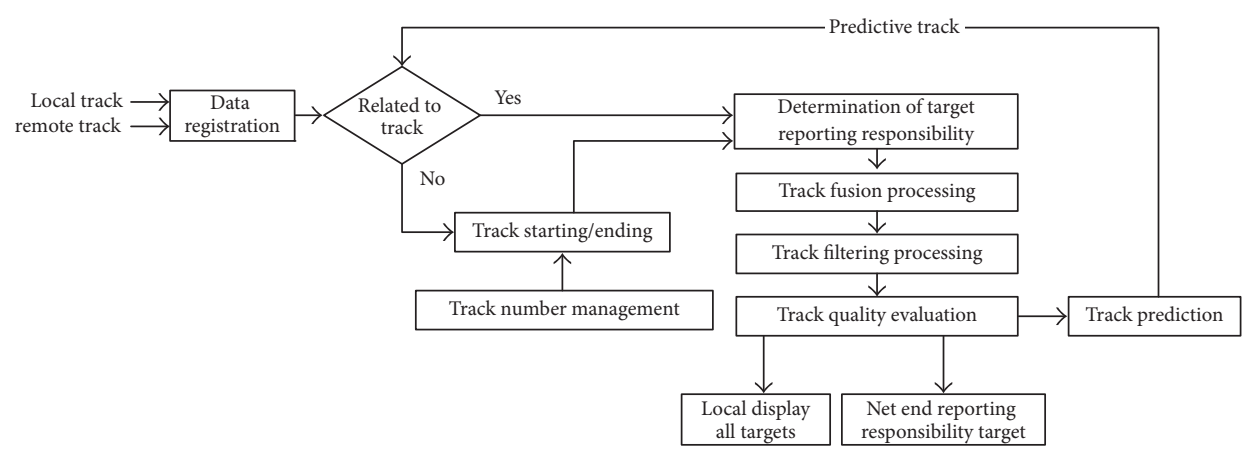

FIGURE 2: Distributed track processing model.

service layer. Information can be fetched from the shared information pool as required by platform application layer, so as to achieve coherence and communion of the situation picture among the whole numbers of data link network [16, 17]. The system architecture is shown in Figure 1.

\section{Distributed Track Processing Model and Method}

Data link distributed track processing is performed by the service layer track fusion processing units on all platforms of the sensor subnet, by following consistent track quality evaluation method and dynamically undertaking target reporting responsibility. The distributed track processing model is shown in Figure 2. Reporting responsibility is used for ensuring that the track reported in the network only comes from the platform which has the best target track information $[18,19]$. The track processing follows three basic principles. (1) First discover first report. That is to say, reporting responsibility is taken by the platform that first detected or initiated target tracking, and a track number from the track number blocks allocated to this platform will be assigned to this target. (2) The best track is reported based on track quality. Namely, if the track quality (TQ1) of one local target held by a platform is higher than the track quality (TQ2) of same target received from a network remote platform (TQ1 - TQ2 $\geqq$ 2 ), then the reporting responsibility of this public track will be taken actively by this local platform, and the existing track number will remain unchanged. (3) Complete report. If a platform holding local track does not receive any track of the same target from network remote platforms over a period of time (e.g., $12 \mathrm{~s}$ ), then the reporting responsibility will be taken over by this platform and the existing track number will remain unchanged. 
Track quality is the key to maintain the correctness and order of reporting responsibility. It is determined by the platform that reports the track, as well as the measurement of the reliability of the reported track position information [20, 21]. The reliability of the position information is represented by "positional accuracy" associated with each TQ value. The level of positional accuracy is defined as a horizontal circular area where the target is actually located with a probability of 0.95 at the time of report. To unify the evaluation standard of track quality for all platforms, target track quality is classified based on the moving object characteristics and the accuracy of typical radar/reconnaissance equipment in the three-dimensional space. With reference to Link 16 criteria, the grading table and corresponding threshold of target track quality in the air, at the sea and on the land is shown in Table 1.

Track quality grading criteria is formed based on target detection accuracy and risk requirement. The threshold of error circle radius of TQ grading from grade 15 to grade 10 is relatively small and the variation rule is twofold increase (0.003 0.096). From grade 10 to grade 9 , the threshold of error circle radius jumps from 0.096 to 0.6 , and the threshold of error circle radius in grades 9 and 8 increases and presents a double relation $(0.6,1.2)$. From grade 8 to grade 7 , the threshold of error circle radius jumps from 1.2 to 3.0. The threshold of error circle radius of TQ grading from grade 7 to grade 3 is larger, presenting equal-difference increase (3.0 to 15.0). The threshold of error circle radius jumps from 15.0 at grades 3 to 30 and 40 at grades 2 and 1 . When the threshold of error circle radius of TQ grading exceeds 40, it indicates non-real-time track. The four different stages of initial value and variation rule of track quality are corresponding to the TQ of aerial targets detected by high accuracy sensors, the TQ of airborne or sea targets gained from relatively high accuracy sensors, and the TQ of moving targets on the sea or land gained from relatively low accuracy sensors.

\section{Track Quality Evaluation Method}

From the existing literatures and relevant application scenarios, the evaluation of track quality mostly adopts the method of $6 \mathrm{D}$ independent measurement target tracking confidence, which is based on the rectangular coordinates position $(x, y, z)$ and speed $\left(v_{x}, v_{y}, v_{z}\right)$ of target. This method assumes 6D measurement mutually independent as premise. Therefore, it takes the spatial covariance and speed covariance of observation error as zero. It is perhaps appropriate for rectangular coordinates target tracking system. For the sensors like radar and reconnaissance working in polar coordinates, the measured 6D parameters of targets are position $(\rho, \alpha, \theta)$ and speed $\left(\nu_{\rho}, v_{\theta}, \nu_{\alpha}\right)$ in polar coordinates, which are only uncorrelated in polar coordinates. If track quality evaluation is conducted in the rectangular coordinates, then the measured values should be converted to rectangular coordinates first of all, which makes the $6 \mathrm{D}$ parameters in the rectangular coordinates relevant, meaning that none of $\left(\sigma_{x y}^{2}, \sigma_{y z}^{2}, \sigma_{z x}^{2} \cdots \sigma_{u v}^{2}, \sigma_{v w}^{2}, \sigma_{w u}^{2}\right)$ is zero. If the independent
TABLE 1: Target track quality (TQ) grading (System).

(a)

\begin{tabular}{lc}
\hline Area $S=\pi r^{2}\left(\mathrm{DM}^{2}\right)$ & TQ grade \\
\hline Non-real-time track & 0 \\
TQ value $>2755$ & 1 \\
TQ value $<2755$ & 2 \\
TQ value $<686$ & 3 \\
TQ value $<439$ & 4 \\
TQ value $<247$ & 5 \\
TQ value $<110$ & 6 \\
TQ value $<27.0$ & 7 \\
TQ value $<4.4$ & 8 \\
TQ value $<1.10$ & 9 \\
TQ value $<0.0281$ & 10 \\
TQ value $<0.0070$ & 11 \\
TQ value $<0.0018$ & 12 \\
TQ value $<0.0004$ & 13 \\
TQ value $<0.0001$ & 14 \\
TQ value $<0.00003$ & 15 \\
\hline
\end{tabular}

(b)

\begin{tabular}{lc}
\hline Radius $r(\mathrm{DM})$ & TQ grade \\
\hline TQ value $>40$ & 0 \\
TQ value $\epsilon(30,40)$ & 1 \\
TQ value $\epsilon(15,30)$ & 2 \\
TQ value $\epsilon(12,15)$ & 3 \\
TQ value $\epsilon(9,12)$ & 4 \\
TQ value $\epsilon(6,9)$ & 5 \\
TQ value $\epsilon(3,6)$ & 6 \\
TQ value $\epsilon(1.2,3)$ & 7 \\
TQ value $\epsilon(0.6,1.2)$ & 8 \\
TQ value $\epsilon(0.096,0.6)$ & 9 \\
TQ value $\epsilon(0.048,0.096)$ & 10 \\
TQ value $\epsilon(0.024,0.048)$ & 11 \\
TQ value $\epsilon(0.012,0.024)$ & 12 \\
TQ value $\epsilon(0.006,0.012)$ & 13 \\
TQ value $\epsilon(0.003,0.006)$ & 14 \\
TQ value $\epsilon(0,0.003)$ & 15 \\
\hline
\end{tabular}

measurement evaluation method is still adopted by ignoring those parameters as zero (actually it is hard to acquire them), then the confidence will be severely affected. This paper proposes a new multisource fusion TQ evaluation algorithm by considering the target-detection-characteristics of sensors.

4.1. Target Tracking Track Quality. During the target track existence, the TQ value is dynamically determined by the state of motion of the target. According to the definition of track quality, the key of calculating track positional accuracy is the determination of correlation gates. Because of the randomness existing in the measurement accuracy of sensors, 
predictive accuracy of the track, and the maneuvering of the target, statistical decision theory is adopted to conduct analysis.

The decision of whether a target point is associated with a track can be seen as the testing of the following opposite hypotheses:

$\mathrm{H}_{0}$ represents the target point and the predictive track does not belong to one target.

$\mathrm{H}_{1}$ represents the target point and the predictive track belongs to one target.

To realize the above-mentioned testing, we need to compare the relative likelihood ratio of $\mathrm{H}_{0}$ and $\mathrm{H}_{1}$ with certain decision threshold [22-25]. For this purpose, let the appropriate interval between the target point and the track as $v$ and calculate the probability of the appearance of $v$ value in every hypothesis, denoted as $p_{0}(v)$ and $p_{1}(\nu)$. In this way, effective testing is as follows:

$$
L(\nu) \triangleq \frac{p_{1}(\nu)}{p_{0}(\nu)} \gtrless \lambda .
$$

Giving all previous measurement values of the $k$ th radar scanning, match the target point that has two-dimensional vector $Z_{k+1}$ with the track that has predictive position $\widehat{Z}_{k+1 / k}$ in the $(k+1)$ th radar scanning. The two-dimensional vector $v$ can be defined as

$$
\nu=Z_{k+1}-\widehat{Z}_{k+1 / k}
$$

Due to the factors like radar measurement error, track predictive error, and target maneuvering, the difference value $v$ is not 0 . Assuming $Z_{k+1}$ and $\widehat{Z}_{k+1 / k}$ are Gaussian random processes, the covariance matrices are $R_{k+1}$ and $\widehat{P}_{k+1 / k}$. Then we make the assumption that the target acceleration is the Gaussian random process when the covariance matrix is Q. Then the target point-track difference is the Gaussian distribution random vectors $v$ with the following covariance matrix:

$$
\Sigma_{\nu}=R_{k+1}+\widehat{P}_{k+1 / k}+\frac{Q T^{4}}{4} .
$$

In the $\mathrm{H}_{1}$ hypothesis, the mean value of $v$ is 0 and the probability density is as follows:

$$
p_{1}(\nu)=\frac{1}{\left(2 \pi\left|\Sigma_{\nu}\right|\right)^{1 / 2}} \exp \left\{-\frac{1}{2} \nu^{T} \Sigma_{\nu}^{-1} \nu\right\} .
$$

If there is no prior information available for the probability distribution of false point, then $p_{0}(\nu)$ could be assumed to be constant. Therefore, in order to carry out this testing, statistical interval $y$ needs to be calculated and then compared with threshold $\chi^{2}$.

$$
y \triangleq v^{T} \Sigma_{\nu}{ }^{-1} v \leq \chi^{2}
$$

$y$ is a random variable with two-dimensional freedom $\chi^{2}$ probability distribution and $\chi$ is an optional parameter, which is used to acquire the assignment of correct association probability and false association probability.

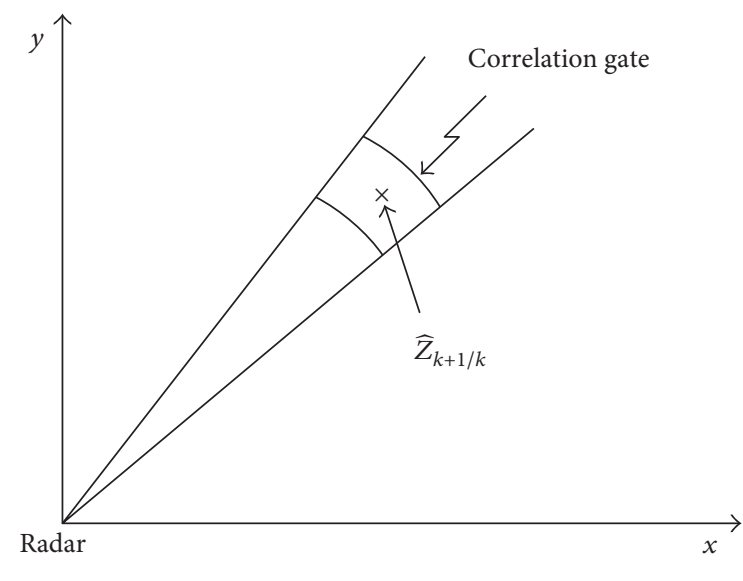

FIgURE 3: Shape of correlation gate.

Correlation is conducted in polar coordinates; then the covariance matrix acquired from (3) formula is

$$
\begin{aligned}
\Sigma_{\nu}= & {\left[\begin{array}{cc}
\sigma_{\rho}^{2} & 0 \\
0 & \sigma_{\theta}^{2}
\end{array}\right]+\left[\begin{array}{cc}
\hat{\sigma}_{\rho, k / k+1}^{2} & 0 \\
0 & \hat{\sigma}_{\theta, k / k+1}^{2}
\end{array}\right] } \\
& +\frac{T^{4}}{4}\left[\begin{array}{cc}
\sigma_{a, \rho}^{2} & 0 \\
0 & \sigma_{a, \theta}^{2}
\end{array}\right] .
\end{aligned}
$$

$\sigma_{a, \rho}^{2}$ and $\sigma_{a, \theta}^{2}$ are the variance of the target acceleration component. To make it simple, let that target acceleration components be not correlative to each other. Substituting formula (6) into formula (5), we obtain the following scalar inequations:

$$
\begin{aligned}
& \left|\rho_{k+1}-\widehat{\rho}_{k+1 / k}\right|<K_{\rho} \\
& \left|\theta_{k+1}-\widehat{\theta}_{k+1 / k}\right|<K_{\theta} .
\end{aligned}
$$

In the inequation, $\left(\rho_{k+1}, \theta_{k+1}\right)$ is the measurement value of point provided by the radar and $\left(\widehat{\rho}_{k+1 / k}, \hat{\theta}_{k+1 / k}\right)$ is the track predictive value of filtering processing. Therefore, the parameters of correlation gates in polar coordinates are as follows:

$$
\begin{aligned}
& K_{\rho}=\chi\left(\sigma_{\rho}^{2}+\widehat{\sigma}_{\rho, k / k+1}^{2}+\frac{T^{4}}{4} \sigma_{a, \rho}^{2}\right)^{1 / 2} \\
& K_{\theta}=\chi\left(\sigma_{\theta}^{2}+\widehat{\sigma}_{\theta, k / k+1}^{2}+\frac{T^{4}}{4} \sigma_{a, \theta}^{2}\right)^{1 / 2} .
\end{aligned}
$$

The shape of correlation gates is shown in Figure 3. We obtain (9) from the target tracking filter algorithm and similar formula in azimuth direction $(\theta)$.

$$
\frac{\widehat{\sigma}_{\rho, k / k+1}^{2}}{\sigma_{\rho}^{2}}=\frac{2(2 k-1)}{k(k+1)} \quad(k \geq 3) .
$$


Substitute formula (9) into formula (8); we obtain the following equations:

$$
\begin{aligned}
& K_{\rho}=\chi\left(1+\frac{2(2 k-1)}{k(k+1)}+\frac{\sigma_{a, \rho}^{2} T^{4}}{4 \sigma_{\rho}^{2}}\right)^{1 / 2} \sigma_{\rho} \\
& K_{\theta}=\chi\left(1+\frac{2(2 k-1)}{k(k+1)}+\frac{\sigma_{a, \theta}^{2} T^{4}}{4 \sigma_{\theta}^{2}}\right)^{1 / 2} \sigma_{\theta} .
\end{aligned}
$$

This has shown that the dimension of correlation gate decreases with the increase of processing period $k$. The more $k$ is accumulated, the higher the reliability of estimation. The steady-state value of correlation gate dimension is associated with the acceleration variance of the target. If the correlation gate directs at the target whose displacement is proportional to $\sigma_{a} T^{2} / 2$, then even highly maneuvering target can be stably tracked. The turning, dodging maneuvering, and acceleration caused by the change of surroundings can all be seen as disturbance to the track. The correlation gates dimension is associated with target type. The correlation gates of the fixed target depend solely on the measurement accuracy. The correlation gates of uniform motion target are calculated based on measurement value and the accuracy of predictive filter while the calculation of the correlation gates of flexible target needs to take acceleration into consideration.

From formula (10), the error area of target track is

$$
S_{r}=\rho K_{\rho} K_{\theta}=\chi^{2}\left(1+\frac{2(2 k-1)}{k(k+1)}+\frac{\sigma_{a}^{2} T^{4}}{4 \rho \sigma_{\rho} \sigma_{\theta}}\right) \rho \sigma_{\rho} \sigma_{\theta}
$$

According to the definition of track quality, the track positional accuracy associated with TQ value falls into the area with a probability of 0.95 . Therefore, let $\chi_{0.05,2}^{2}=5.991$, and calculate the target track error $S_{r}$ in each scanning period $k$; then we can determine the TQ value of each track point during single radar track tracking process by referring to Table 1(a).

In general sense, distributed target track fusion is multisource fusion. The sources include point or track acquired by one or several sensors of this platform and the target track reported by far-end platform from the network. When several sensors detect the same target, track processing error can be seen as the accumulation of several single radar correlation gates. The track error function after accumulation is 2-D normal random function. To simplify calculation, correlation gates shape can be approximately considered as circle, as is shown in Figure 4. In this way, multisource track processing error area can be expressed as

$$
\begin{aligned}
S_{m} & =\frac{\pi}{4}\left(K_{\rho}^{2}+\rho^{2} K_{\theta}^{2}\right) \\
& =\frac{\pi \chi^{2}}{4}\left(\left(1+\frac{2(2 k-1)}{k(k+1)}\right)\left(\sigma_{\rho}^{2}+\rho^{2} \sigma_{\theta}^{2}\right)+\frac{T^{4}}{4} \sigma_{a}^{2}\right) .
\end{aligned}
$$

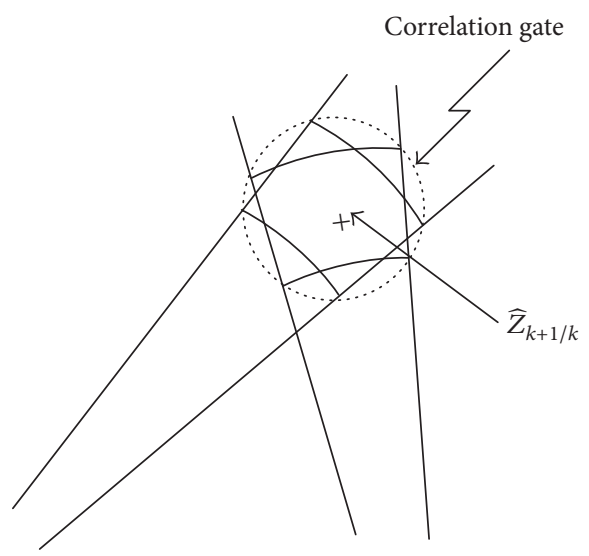

FIGURE 4: Multisource track fusion correlation gate shape.

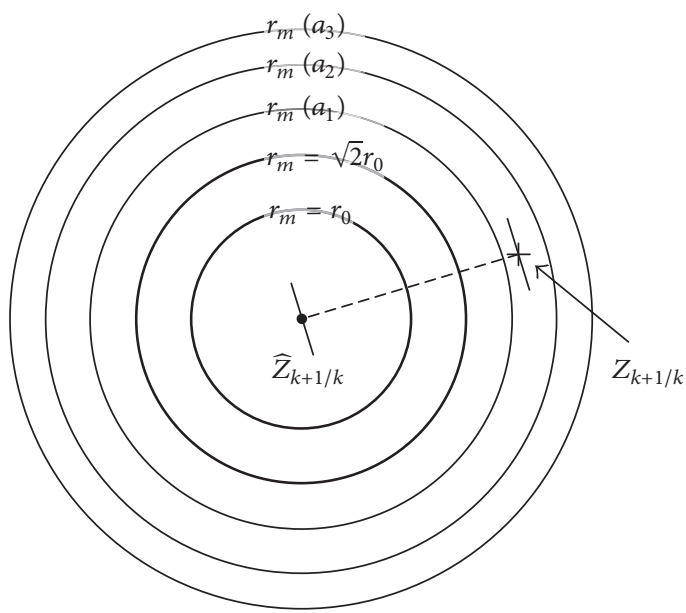

FIGURE 5: Target track correlation gate diagrammatic.

Assume $\left(\chi^{2} / 4\right)\left(\sigma_{\rho}^{2}+\rho^{2} \sigma_{\theta}^{2}\right)=r_{0}^{2}$ and $\chi_{0.05,2}^{2}=5.991$; then it is converted to the distance data $r_{m}$ characterized track quality. We will have the following relational expression:

$$
r_{m}^{2}=r_{0}^{2}+\frac{2(2 k-1)}{k(k+1)} r_{0}^{2}+\frac{\chi^{2} T^{4}}{16} \sigma_{a}^{2} \quad(k \geq 3)
$$

According to $r_{m}$ and Table 1(b), we can determine the TQ value of each track point during the tracking process.

We can know from formula (13) for systems where sensors are defined that the first item mainly varies along with the change of target distance, which is the basic parameter for determining the track quality; the second item represents the track quality of moving targets and is also associated with tracking filter parameters; the third item is the influence brought by the maneuvering of targets or acceleration, showing random characteristics. In the process of track processing, based on target classification, a group of acceleration $a_{i}$ can be estimated and the additional value of correlation gates radius can be calculated, used to capture the maneuvering of the target, as shown in Figure 5. 
When the measurement point of the target falls into certain additional area of the correlation gates, it means that the target is generating maneuvering or acceleration. Therefore, we can estimate the acceleration range of the tracking target to further determine the change of track quality. Meanwhile, appropriate adjustment of tracking filter parameters can be made through adjustment of $k$ value, achieving the balance between track smoothness and fast track.

4.2. Target Initiation Track Quality. The target initiation track is defined as a track phase from the first target point to be turned into the reliable track. The initiation track quality is mainly determined by the scale of track initiation correlation gates.

Let $z$ be the target position measurement value $(\rho, \theta)$. The measurement value $z$ is disturbed by the steady random error $w$ with zero-mean value and covariance matrix $R$. $V$ represents the target speed component $(\dot{\rho}, \dot{\theta})$. Because of the lack of track information during the track initiation phase, we can assume that $V$ is the random vector with zero-mean value and covariance matrix $\Sigma_{V}$. The target displacement $d$ of two successive points can be exported as

$$
d=Z_{k+1}-Z_{k}=V T+w_{k+1}-w_{k}
$$

$d$ is also the zero-mean random vector with the following covariance matrix:

$$
\Sigma_{d}=\Sigma_{V} T^{2}+2 R
$$

To judge whether the two successive points belong to the same target, the following testing needs to be conducted:

$$
y \triangleq d^{T} \Sigma_{d}^{-1} d \leq \chi^{2}
$$

$y$ is the random vector with 2-D freedom $\chi^{2}$ probability distribution. It can be written as using formula (15):

$$
\Sigma_{d}=\left[\begin{array}{cc}
\sigma_{\dot{\rho}}^{2} & 0 \\
0 & \sigma_{\dot{\theta}}^{2}
\end{array}\right] T^{2}+2\left[\begin{array}{cc}
\sigma_{\rho}^{2} & 0 \\
0 & \sigma_{\theta}^{2}
\end{array}\right] .
$$

In polar coordinates, the velocity vectors of $\rho$ and $\theta$ direction are uncorrelated and the variances, respectively, are $\sigma_{\dot{\rho}}^{2}, \sigma_{\dot{\theta}}^{2}$. Formula (15) can be decomposed into the following scalar relations:

$$
\begin{aligned}
& \left|\rho_{k+1}-\rho_{k}\right|=\frac{\chi}{2} \sqrt{\sigma_{\dot{\rho}}^{2} T^{2}+2 \sigma_{\rho}^{2}} \\
& \left|\theta_{k+1}-\theta_{k}\right|=\frac{\chi}{2} \sqrt{\sigma_{\dot{\theta}}^{2} T^{2}+2 \sigma_{\theta}^{2}}
\end{aligned}
$$

We can see that the shape of track initiation correlation gates is the same as the correlation gates of point-track during the track tracking process. In this formula, $T$ is the time interval of the two successive points. Limitation $\Delta \theta$ should be imposed when the distance between the target and the sensor is small to avoid excessive value. To simplify the processing, the correlation gates in formula (18) can be approximate to circle considering the multisource fusion. In this way, the track initiation error area is

$$
\begin{aligned}
S_{m} & =\pi\left(\left|\rho_{k+1}-\rho_{k}\right|^{2}+\rho^{2}\left|\theta_{k+1}-\theta_{k}\right|^{2}\right) \\
& =\frac{\pi \chi^{2}}{4}\left(2\left(\sigma_{\rho}^{2}+\rho^{2} \sigma_{\theta}^{2}\right)+\sigma_{\nu}^{2} T^{2}\right),
\end{aligned}
$$

where $\sigma_{v}^{2}=\sigma_{\dot{\rho}}^{2}+\rho^{2} \sigma_{\dot{\theta}}^{2}$

$$
\text { Assume }\left(\chi^{2} / 4\right)\left(\sigma_{\rho}^{2}+\rho^{2} \sigma_{\theta}^{2}\right)=r_{0}^{2} \text { and } \chi_{0.05,2}^{2}=5.991 \text {, and }
$$
then it is converted to the distance data $r_{m}$ representing track quality. We will have the following relations:

$$
r_{m}^{2}=2 r_{0}^{2}+\frac{\chi^{2} T^{2}}{4} \sigma_{\nu}^{2}
$$

According to $r_{m}$ and Table 1(b), TQ value of each track point during track initiation period can be determined. During track initiation period, track quality is not related to tracking filter parameters.

4.3. Typical Track Quality Curve. We can see that track quality is associated with the detection performance of sensors from the above-mentioned track quality model and algorithm. The higher the measurement accuracy and the data rate, the greater the TQ value. Track quality is associated with tracking filter parameters. The more stable the track, the higher the TQ value. Track quality is associated with the distance between the target and the sensor. The shorter the distance, the higher the TQ value. Track quality is associated with the maneuverability of the target. The greater the maneuverability, the greater the change of TQ value. Initial track quality is also associated with target speed.

The influence of sensor detection performance on track quality is analyzed through computer simulation. Suppose distributed track fusion is conducted by one data link sensor subnet, forming target situation picture that can be shared in real time in the whole network, where the measurement accuracy of radar on certain platform is $\sigma_{\rho}=100 \mathrm{~m}, \sigma_{\theta}=$ $0.10^{\circ}$ and its scanning period is $T=4 \mathrm{~s}$. If the target speed is $300 \mathrm{~m} / \mathrm{s}$ and the influence of target distance on TQ is not considered (let $\rho=100 \mathrm{~km}$ ), the TQ value change for various states like target track initiation, rectilinear motion, and maneuvering $\left(a=10 \sim 80 \mathrm{~m} / \mathrm{s}^{2}\right)$ can be acquired according to formulas (13) and (20). As shown in Figure 6(1), the TQ value varies between 8 and 9 and the maneuvering of the target has no impact on TQ value. If the measurement accuracy of radar is $\sigma_{\rho}=300 \mathrm{~m}, \sigma_{\theta}=0.25^{\circ}$, the scanning period is $T=10 \mathrm{~s}$, and other conditions remain unchanged, then we find that the track quality declines during track initiation and target maneuvering period and the TQ value varies between 7 and 9, as shown in Figure 622. If the measurement accuracy of several sensors on the platform is different, we need two steps to obtain the track quality while tracking the same target. First, acquire a comprehensive measurement accuracy value through a weighting function of the measurement accuracy of the sensors. Second, conduct the same algorithm with the comprehensive value. 


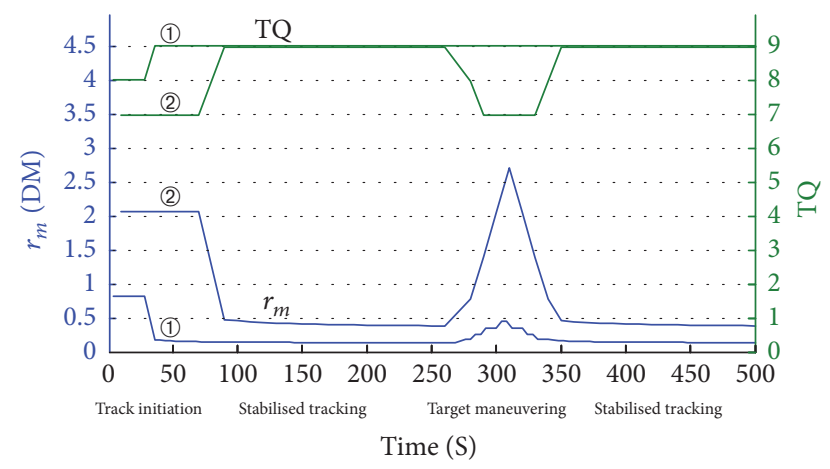

FIGURE 6: Relationship between TQ and sensor performancetracking filtering.

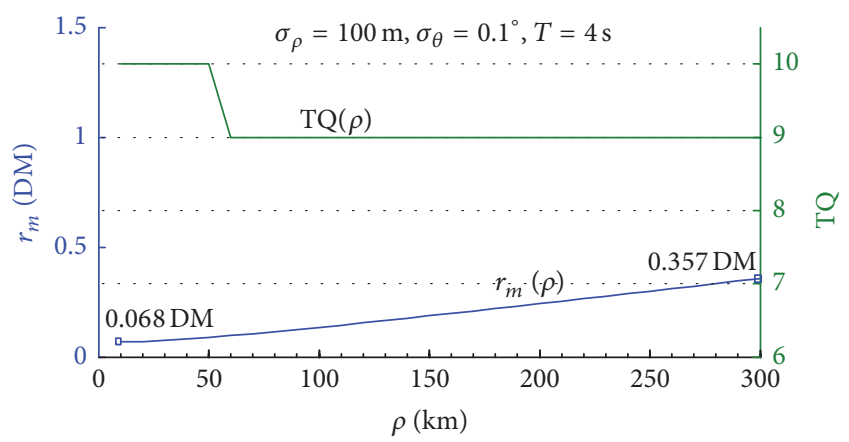

FIGURE 7: Relationship between TQ and target distance-tracking filtering.

The following explains the impact of the change of target distance on track quality through computer simulation. Let the radar measurement accuracy be $\sigma_{\rho}=100 \mathrm{~m}, \sigma_{\theta}=$ $0.10^{\circ}, T=4 \mathrm{~s}$, and the radar stably track targets moving at a constant speed in the range of 10 to $300 \mathrm{~km}$. The TQ value will decrease from 10 to 9, as shown in Figure 7.

\section{Conclusion}

The key in distributed target track fusion is network reporting responsibility management and track quality evaluation algorithm. This paper introduced the distributed track reporting mechanism of "first discover first report, quality-prioritybased report and succeed report," and proposed a new track quality evaluation algorithm. The proposed algorithm is well matched with the convergence process of tracking filter. It avoids the target tracking confidence problem caused by the difficulty in acquiring correlation information when applying the target tracking confidence method in the rectangular coordinates. The track quality evaluation algorithm has good reliability, timeliness, and practicability, which can generate TQ value simultaneously during track fusion processing in practical application. The typical track quality curve via computer simulation indicates that this algorithm has good continuity and value rationality in track initiation, tracking, and target maneuvering.

\section{Conflicts of Interest}

The authors declare that they have no conflicts of interest.

\section{Acknowledgments}

This research was supported by National Natural Science Foundation of China (Grant no. 60939003) and partly supported by Natural Science Fund Project in Jiangsu Province (BK2011737). The authors would like to thank them for their financial support.

\section{References}

[1] N. Friedman, Network Centric Warfare: How Navies Learned to Fight Smarter through Three World Wars, Naval Institute Press, Annapolis, Md, USA, 2009.

[2] X. Zhang, Y. Liang, Z. Wang, and F. Yang, "Joint estimation of target state and ionosphere state of over-the-horizon radar," in Proceedings of the 16th International Conference on IEEE Information Fusion (FUSION '13), pp. 1066-1071, Istanbul, Turkey, July 2013.

[3] M. G. Rutten, S. Maskell, M. Briers, and N. J. Gordon, "Multipath track association for over-the-horizon radar using lagrangian relaxation," in Proceedings of the Signal and Data Processing of Small Targets 2004, vol. 5428 of Proceedings of SPIE, pp. 452-463, Orlando, Fla, USA, April 2004.

[4] T. Sathyan, T.-J. Chin, S. Arulampalam, and D. Suter, "A multiple hypothesis tracker for multi-target tracking with multiple simultaneous measurements," IEEE Journal on Selected Topics in Signal Processing, vol. 7, no. 3, pp. 448-460, 2013.

[5] V. K. Saini and H. B. Hablani, "Air-to-air tracking performance with inertial navigation and gimballed radar: a kinematic scenario," in Proceedings of the 3rd International Conference on Advances in Control and Optimization of Dynamical Systems (ACODS '14), pp. 234-241, March 2014.

[6] B. Raj, Homing missile antenna control [Ph.D. thesis], Indian Institute of Technology, Bombay, India, 2012.

[7] A. J. Shah, Level flight control, laws for F-16 aircraft [M.S. thesis], Indian Institute of Technology, Bombay, India, 2013.

[8] H. B. Hablani, "Autonomous inertial relative navigation with sight-line-stabilized integrated sensors for spacecraft rendezvous," Journal of Guidance, Control, and Dynamics, vol. 32, no. 1, pp. 172-183, 2009.

[9] R. M. Rogers, Applied Mathematics in Integrated Navigation Systems, chapter 1, 2, 6, AIAA, Reston, Va, USA, 2003.

[10] Y. Bar-Shalom, X. Li, and T. Kirubarajan, Estimation with Applications to Tracking and Navigation, John Wiley \& Sons, Inc., New York, NY, USA, 2001.

[11] K. Sun and X. Chen, "Sky-wave OTHR data association method based on track clustering," Modern Radar, vol. 33, no. 10, pp. 5159, 2011.

[12] H.-X. Liu, Y. Liang, X.-Y. Chen, Q. Pan, and F. Yang, "An adaptive OTHR multipath track fusion algorithm," Acta Electronica Sinica, vol. 37, no. 6, pp. 1348-1352, 2009.

[13] Y. Zhang, Principle of Radar Electronic Warfare, National Defense Industry Press, Beijing, China, 2006.

[14] S. Liu, Z. Xing, and P. Zhao, "Present situation and development trend of radar counter countermeasures technology," Ship Electronic Engineering, vol. 31, no. 8, pp. 11-13, 2011. 
[15] Y. Li, "Tactical data link and its application technology," Journal of China Academy of Electronics and Information Technology, vol. 2, pp. 211-217, 2007.

[16] Y. Li and Y. Mao, "Research on design method of data link tactical function," Journal of China Academy of Electronics and Information Technology, vol. 9, pp. 453-457, 2014.

[17] Y. Li, "Integrated application technology of multifarious data links," Modern Electronic Engineering, vol. 11, pp. 72-75, 2008.

[18] Department of Defense Interface Standard, “Tactical Data Link (TDL) 16 message standard,” Tech. Rep. MIL-STD-6016D, 2008.

[19] K. J. Held and B. H. Robinson, "TIER II Plus airborne EO sensor LOS control and image geolocation," in Proceedings of the IEEE Aerospace Conference, pp. 377-405, IEEE, Snowmass at Aspen, Colo, USA, February 1997.

[20] G. Biernson, Optimal Radar Tracking Systems, Wiley, New York, NY, USA, 1990.

[21] R. S. Baheti, "Efficient approximation of kalman filter for target tracking," IEEE Transactions on Aerospace and Electronic Systems, vol. 22, no. 1, pp. 8-14, 1986.

[22] A. Farina and F. A. Studer, Radar Data Processing (Volume I), Research Studies Press LTD, Baldock, UK, 1985.

[23] A. Farina and F. A. Studer, Radar Data Processing, Volume II, Research Studies Press, Baldock, UK, 1985.

[24] S. B. Colegrove and S. J. Davey, "PDAF with multiple clutter regions and target models," IEEE Transactions on Aerospace and Electronic Systems, vol. 39, no. 1, pp. 110-124, 2003.

[25] Y. Bar-Shalom and D. B. William, Multitarget-Multisensor Tracking, Applications and Advances, Artech House, New York, NY, USA, 2001. 


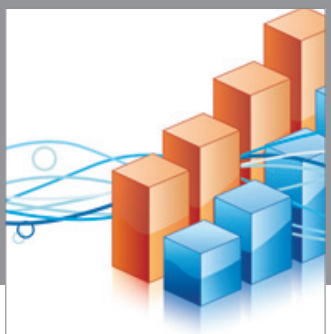

Advances in

Operations Research

vatem alat4

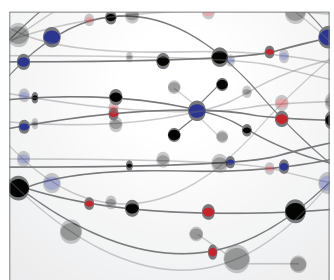

\section{The Scientific} World Journal
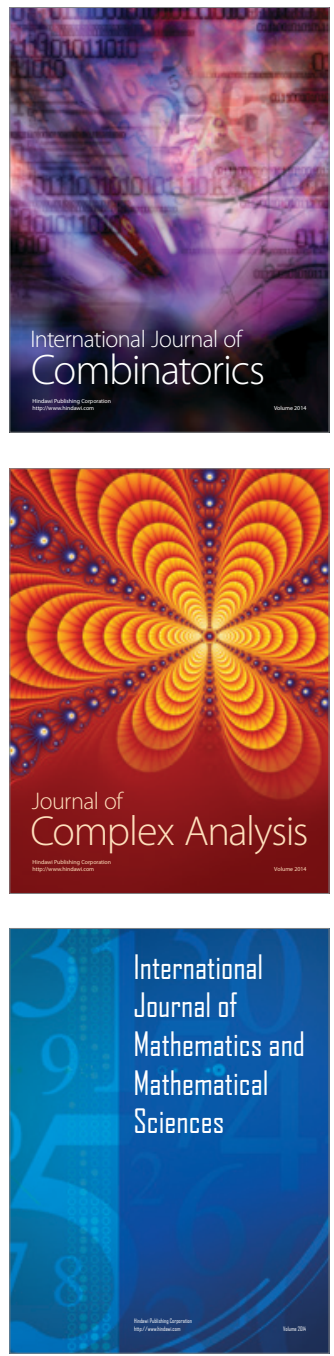
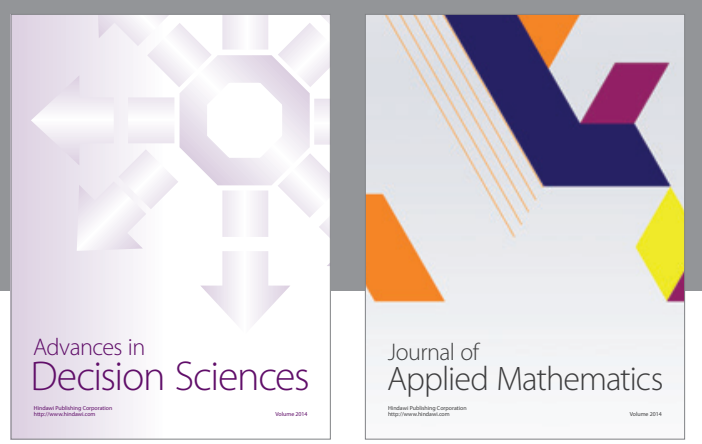

Algebra

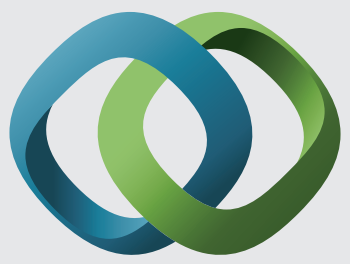

\section{Hindawi}

Submit your manuscripts at

https://www.hindawi.com
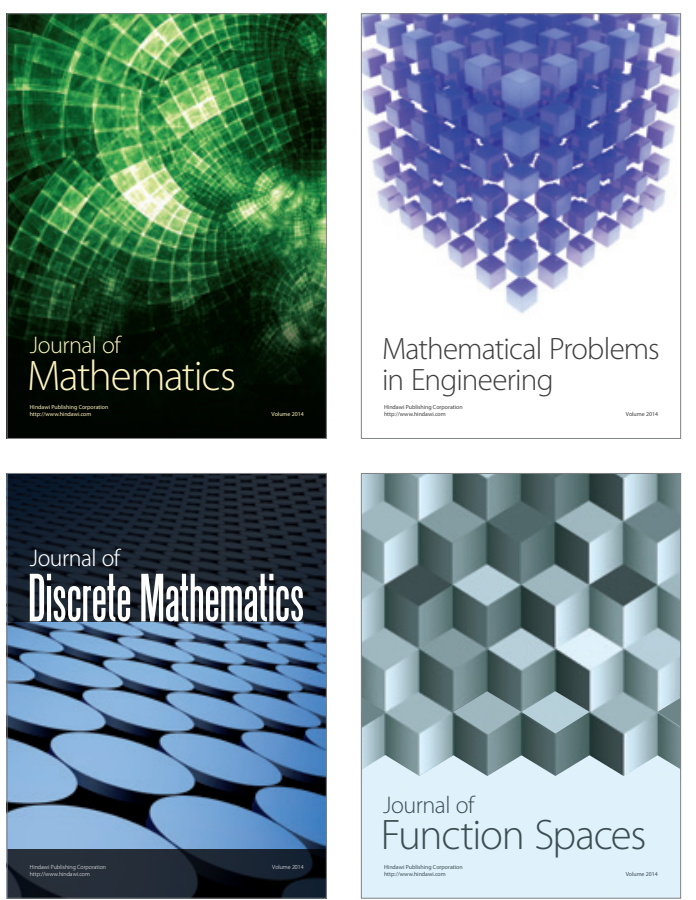

Mathematical Problems in Engineering
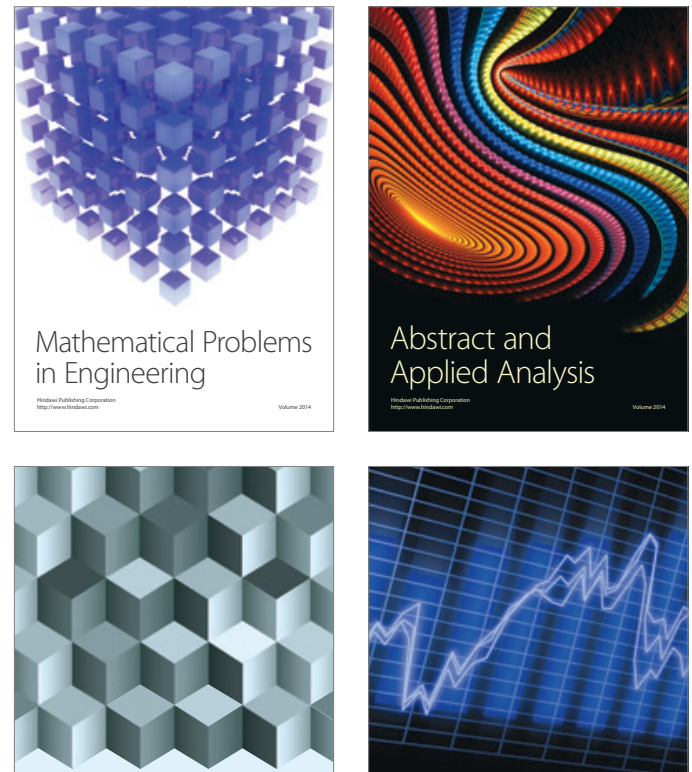

Journal of

Function Spaces

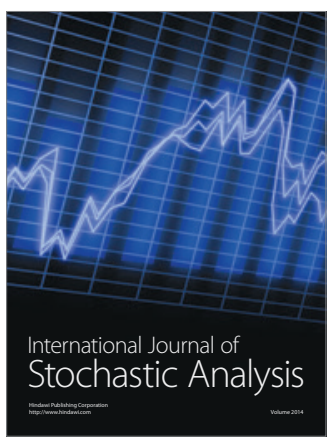

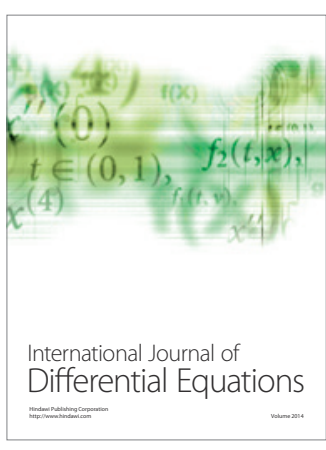
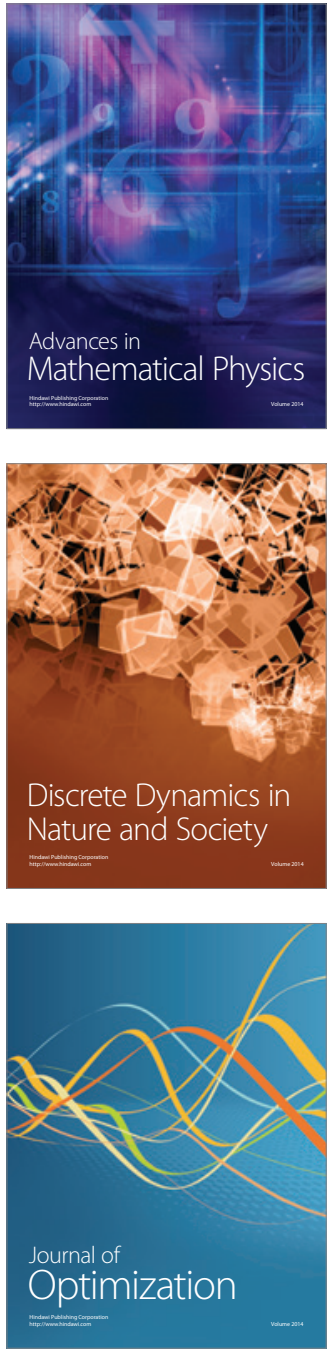